\title{
Behavior of Fluoropolymers in Presence of Tritiated Water
}

\author{
G. Bubueanu*, C. Postolache, V. Fugaru, C. Tuta \\ Horia Hulubei National Institute for Physics and Nuclear Engineering, 30, Reactorului St., Magurele, Romania

\begin{abstract}
The behaviour of Polytetrafluoroethylene (PTFE), NAFION ionomer membranes and VITON fluoroelastomer in presence of high activity tritiated water (HTO) was analysed. Radiolytic stability of the fluoropolymers-HTO system was analysed by: (a) Simulation of radiolytic processes by quantum mechanical methods, (b) Simulation of radiolytic effects by exposure of fluoropolymers immersed in $\mathrm{H}_{2} \mathrm{O}$ to ${ }^{60}$ Co gamma radiation field, (c) Immersing of fluoropolymers samples in HTO with high activity, for different time periods. In both cases the samples were characterized by FT IR ATR, and study of emission of fluoride ions from polymer matrix.
\end{abstract}

DOI: 10.12693/APhysPolA.127.1363

PACS: $61.82 . \mathrm{Pv}$

\section{Introduction}

One of the most used methods for tritium recovery from the tritiated water (HTO) or from tritiated heavy water relies on the combined techniques of Combined Electrolysis Catalytic Exchange and Cryogenic Distillation processes [1]. The electrolytic cell converts the tritiated water or tritiated heavy water into a mixture of hydrogen, deuterium and tritium. The mixture is purified and then tritium is recovered by catalytic isotopic separation technique [2]. Tritium presence in the system induces an intense radiation field, especially at the interface between the polymeric support and the aqueous solution. Significant structural alterations of fluoropolymers with potentially negative effects can be identified. In this paperwork the radiolytic effects induced by the presence of tritiated water / heavy water were studied.

\section{Materials and methods}

\subsection{Simulation of radiolytic effects by quantum mechanical method}

For simulation of polymeric substrate behaviour in the ionizing radiation field, in this study, olygomeric structures with molecular masses between 1000 and 2500 Dalton were used. The simulation was realized on Intel Duo Core T6600 computer with 3 GB DD RAM using HYPERCHEM 4 and 7 computing software.

For evaluation of primary radiolytic effects, an original model [3] was used, which includes: (a) ionization of molecules by the electron release from highest occupied molecular orbital, followed by molecule rearrangement for reaching the minimum energy value; (b) molecule neutralization by a free electron, captured from medium, and fast molecule fragmentation without geometrical reoptimization. The chemical bonds predisposed to breakage due to radiolysis have a minimum binding energy (BE). It is necessary to analyze the spatial distribution

* corresponding author; e-mail: gbubueanu@nipne.ro of molecular orbital to identify the molecule zones where energy absorption, respectively electron capture by ionizing molecule is happening. BE were determined by simulation of homolytic dissociation processes [4].

Radiochemical yields $G$ were associated with the probability of homolytic fragmentation of chemical bonds. In this sense $G$ represents the sum of individualized yields $G_{i}$ for every chemical bond which is present in the area covered by LUMO orbital.

$$
G=\sum G_{i}=\sum a x \frac{E_{\text {med }}}{E_{i}} A_{\mathrm{LUMO}(\mathrm{i})},
$$

where: $a$ is parameterization constant deduced from experimental data, $E_{i}$ is binding energy, $E_{\text {med }}$ is average $\mathrm{BE}$ for coverage area of LUMO orbital; $A_{\mathrm{LUMO}(\mathrm{I})}$ is LUMO orbital coverage degree of the chemical bond, and $x$ is weighting factor for protector effect of delocalized orbital.

\subsection{Evaluation of absorbed doses at the interface fluoropolymers-tritiated water}

Due to the very low energy of $\beta$-radiation emitted by tritium, the direct measurements of absorbed dose by the fluoropolymers, using classical methods is practically impossible. For this purpose an evaluation model was developed. A hemisphere represents the volume of tritiated water that irradiates the polymer surface. The radius of the hemisphere is equal to the maximal rate of $\beta$-radiation emitted by tritium. The flow rate of absorbed dose is computed using relation: $d=\Phi E_{m} / m$, where $d$ is dose flow rate; $\Phi$ is total radiation flux, which interacts with the material surface; $E_{m}$ main energy of tritium $\beta$-radiation and $m$ is the mass of exposed material.

$$
\Phi=\sum_{i=0}^{i=60} \Phi_{i}=\sum_{i=0}^{i=60} \varphi_{i} \alpha_{i} y_{i},
$$

where: $\varphi_{i}$ is the radiation flux emitted by tritium atoms of water volume circumscribed by two hemispheres; $\alpha_{i}$ is the emission coefficient inside of the effective angle (in the direction of spot surface) at the level of semispherical dome; $\mathrm{y}_{i}$ is the coefficient of attenuation in tritiated water stratum of $\beta$ radiation emitted by tritium from water volume circumscribed by two hemispheres and absorbed 
in tritiated water volume circumscribed by internal hemisphere surface.

Based on previously presented data the value of dose rate can be calculated using the formula:

$$
\begin{aligned}
& d=\frac{9.131 \times 37 \times 10^{-22} \pi n}{3 m} \sum_{i=0}^{i=60}\left(3 i^{2}+3 i+1\right) \\
& \times \mathrm{e}^{-i / 5.77078}\left(1-\frac{i}{\sqrt{i^{2}+1}}\right)=n \times 24.25(\mu \mathrm{Gy} / \mathrm{s}) .(3)
\end{aligned}
$$

Using this equation, the value of dose flow rate can be calculated as a function of volume concentration $n$, expressed in GBq/l.

\subsection{Simulation of the tritiated water effects by exposure to gamma radiation field}

The PTFE, VITON and NAFION samples were exposed to ${ }^{60} \mathrm{Co}$ gamma source, which provided a dose rate of $6.67 \mathrm{kGy} / \mathrm{h}$. Samples weighting approximately $500 \mathrm{mg}$ were introduced in $20 \mathrm{ml}$ HDPE vials containing $10 \mathrm{ml}$ of ultra purified water. The conductivity was $0.3 \mu \mathrm{S}$, with Total Organic Carbon (TOC) of 14 ppb. Absorbed doses, correlated with calculated value of HTO exposure, were 300,450 and $1000 \mathrm{kGy}$.

\subsection{The exposure of samples to tritiated water with high activity}

Tritiated water with high activity was obtained by isotope exchange technique ${ }^{3} \mathrm{~T}_{2}-{ }^{3} \mathrm{He}: \mathrm{H}_{2} \mathrm{O}^{4}$. HTO with initial activity of $45 \mathrm{GBq} / \mathrm{ml}$ has been purified by double distillation in quartz device. The dilutions were performed using ultra purified water.

The fluoropolymers weighting approximately $500 \mathrm{mg}$ were introduced in $4 \mathrm{ml} \mathrm{HDPE}$ vials containing $3 \mathrm{ml}$ of $\mathrm{HTO}$ with radioactive concentration of $3.28 \mathrm{TBq} / 1$.

The samples were stored for 60 and 182 days respectively, at room temperature in a glove box. The total concentrations of fluoride ions were measured in tritiated water.

The samples were decontaminated by multiple washing with water and methanol and finally dried by storing for 24 hours in a vacuum desiccator.

\subsection{Evaluation of radiolytic processes by $I R$ spectrometry and fluoride emission}

The radio-induced fluoride release was studied by determination of $\mathrm{pH}$ and $\mathrm{F}^{-}$ions concentration using a WTW INOLAB MULTI $720 \mathrm{pH}$-meter with $\mathrm{F}^{-}$selective electrode [5]. The radiochemical yields were estimated by correlation between the obtained results, sample masses and absorbed doses.

The radio-induced alterations of polymeric structures have been analyzed using IR spectrometry. In this study an FTIR ATR TENSOR 27 spectrometer, Bruker type, was used. The IR spectra for samples irradiated in a $\gamma$ field, for samples exposed to tritiated water and for reference samples were collected and compared.

\section{Results}

\subsection{Evaluation of absorbed dose at the interface: solid surfaces-tritiated water}

For radioactive concentration of $3.28 \mathrm{TBq} / 1$, the calculated dose rate is $79.52 \times 10^{-3} \mathrm{~Gy} / \mathrm{s}$. On the basis of dose rate, the value of $412 \mathrm{kGy}$ is determined for 60 days and value of $1250 \mathrm{kGy}$ is determined respectively for the 182 days exposure to tritiated water.

\subsection{Simulation of radiolytic effects by quantum chemical methods}

In case of PTFE, the simulation of primary radiolytic effect reveals a prominent radio-sensibility of the main polymeric chain. In case of geometrically optimized PTFE structures, in the ionized state C-C BE manifests an important energetic decrease from $268.77 \mathrm{~kJ} / \mathrm{mol}$, for neutral structure, to $24.40 \mathrm{~kJ} / \mathrm{mol}$. Polymeric chain analysis in LUMO orbital zone shows a significant modification of the stereochemistry of $\mathrm{C}$ atoms, so that $\mathrm{C}$ atoms have the tendency of conversion of hybrid state from $\mathrm{sp}^{3}$ to $\mathrm{sp}^{2}$. This fact represents practically the transition state in homolytic fragmentation of polymeric chain at $\mathrm{C}-\mathrm{C}$ bond level.

In case of NAFION structure, modeling of primary radiolytic effect reveals a relative stability of PTFE backbone for those two structures (acid form and salt form). Radio sensibility of main chain is still low, because the energy decrease is not significant and LUMO orbital is distributed in the sulfuric acid terminal zone.

Hydrophilic structure presents reduced radiolysis stability; C-S, C-C and $\mathrm{C}-\mathrm{O}$ are the bonds, which are most exposed to fragmentation. The determined radiochemical yields present high value in a case of PTFE and an inferior value in NAFION case, but they reveal the tendency of losing of the hydrophilic groups.

In VITON case, the simulation of primary radiolytic effects suggests a higher stability with respect to PTFE. LUMO orbital is located on the main chain, at tetrafluoroethylene monomer unit. The $\mathrm{F}_{2} \mathrm{C}-\mathrm{CF}_{2} \mathrm{BE}$ decreases from $269.69 \mathrm{~kJ} / \mathrm{mol}$ (for neutral structure) to $219.91 \mathrm{~kJ} / \mathrm{mol}$ (in transitional state case). Because LUMO orbital covering degree of the analyzed chemical bond has a small value, individualized yields $G_{i}$ are not dominant, like in the PTFE case. Radio sensibility of $\mathrm{C}-\mathrm{F}$ and $\mathrm{C}-\mathrm{CF}_{3}$ bond is still low, because the energy decrease is not significant and LUMO orbital is distributed in $\mathrm{C}-\mathrm{C}$ main chain zone. The values of radiochemical yields obtained by simulation are shown in Table I.

\subsection{Evaluation of radiolytic processes by fluoride emission}

Determination of concentration of fluoride ions in aqueous $\left(\mathrm{H}_{2} \mathrm{O}\right.$ /gamma radiation and HTO) solutions reveals a higher stability of VITON and NAFION with respect to PTFE. The determined values of radiochemical yields for fluoride release $G_{F}$ are shown in Table II. 
Determination of radiochemical yields by

TABLE I computational methods.

\begin{tabular}{c|c|c|c|c}
\hline \hline $\begin{array}{c}\text { Radiochemical } \\
\text { yield }\end{array}$ & $\begin{array}{c}\text { PTFE } \\
\text { PTFE }\end{array}$ & $\begin{array}{c}\text { NAFION } \\
\text { salt form }\end{array}$ & $\begin{array}{c}\text { NAFION } \\
\text { acid form }\end{array}$ & VITON A \\
\hline$G(\mathrm{C}-\mathrm{F})$ & 0.033 & 0.23 & 0.15 & 0.038 \\
$G(\mathrm{C}-\mathrm{C})_{\text {main chain }}$ & 1.230 & 0.25 & 0.22 & 0.093 \\
$G\left({\left.\mathrm{C}-\mathrm{CF}_{3}\right)}\right.$ & & & & 0.004 \\
$G(\mathrm{C}-\mathrm{O})$ & - & 0.23 & 0.22 & \\
$G(\mathrm{C}-\mathrm{S})$ & - & 0.32 & 0.25 & \\
$G(\mathrm{C}-\mathrm{H})$ & & & & 0.004
\end{tabular}

Fluoride radioinduced emissions analysis from PTFE, NAFION and VITON samples.

TABLE II

\begin{tabular}{c|c|c|c|c}
\hline \hline Sample & $\begin{array}{c}\text { Dose } \\
{[\mathrm{kGy}]}\end{array}$ & $\begin{array}{c}\mathrm{F}^{-} \text {conc. } \\
{[\mathrm{mol} / \mathrm{l}]}\end{array}$ & $\begin{array}{c}\text { Release F/ } \\
\text { mass sample }\end{array}$ & $G\left(\mathrm{~F}^{-}\right)$ \\
\hline PTFE: $\mathrm{H}_{2} \mathrm{O}$ & 1000 & $2.33 \times 10^{-2}$ & $4.66 \times 10^{-4}$ & 1.75 \\
PTFE: $\mathrm{H}_{2} \mathrm{O}$ & 450 & $1.67 \times 10^{-2}$ & $3.34 \times 10^{-4}$ & 2.79 \\
PTFE: $\mathrm{H}_{2} \mathrm{O}$ & 300 & $1.32 \times 10^{-2}$ & $2.64 \times 10^{-4}$ & 3.31 \\
PTFE:HTO & 410 & $5.74 \times 10^{-2}$ & $3.44 \times 10^{-4}$ & 3.16 \\
PTFE:HTO & 1250 & $7.64 \times 10^{-2}$ & $4.58 \times 10^{-4}$ & 1.38 \\
NAFION: $\mathrm{H}_{2} \mathrm{O}$ & 1000 & $8.76 \times 10^{-3}$ & $1.75 \times 10^{-4}$ & 0.66 \\
NAFION: $\mathrm{H}_{2} \mathrm{O}$ & 450 & $4.50 \times 10^{-3}$ & $9.00 \times 10^{-5}$ & 0.75 \\
NAFION: $\mathrm{H}_{2} \mathrm{O}$ & 300 & $2.68 \times 10^{-3}$ & $5.36 \times 10^{-5}$ & 0.67 \\
NAFION:HTO & 410 & $1.41 \times 10^{-2}$ & $8.48 \times 10^{-5}$ & 0.78 \\
NAFION:HTO & 1250 & $3.99 \times 10^{-2}$ & $2.39 \times 10^{-4}$ & 0.72 \\
VITON: $\mathrm{H}_{2} \mathrm{O}$ & 1000 & $1.53 \times 10^{-3}$ & $3.07 \times 10^{-5}$ & 0.12 \\
VITON: $\mathrm{H}_{2} \mathrm{O}$ & 450 & $1.12 \times 10^{-3}$ & $2.23 \times 10^{-5}$ & 0.19 \\
VITON: $\mathrm{H}_{2} \mathrm{O}$ & 300 & $9.79 \times 10^{-4}$ & $1.96 \times 10^{-5}$ & 0.25 \\
VITON:HTO & 410 & $4.50 \times 10^{-3}$ & $2.70 \times 10^{-5}$ & 0.25 \\
VITON:HTO & 1250 & $6.60 \times 10^{-3}$ & $3.96 \times 10^{-5}$ & 0.12
\end{tabular}

\subsection{Evaluation of radiolytic processes by IR spectrometry}

In PTFE case, experimental results (spectral analysis in Fig. 1) support the results of simulation. Thus the characteristic bands for unassociated carboxyl $\mathrm{OH}\left(3470 \mathrm{~cm}^{-1}\right)$ and trifluoroacetic acid (TFA) band $\left(1780 \mathrm{~cm}^{-1}, 1440 \mathrm{~cm}^{-1}, 813 \mathrm{~cm}^{-1}\right)$ were identified. In the reference sample, peaks from region of $2930 \mathrm{~cm}^{-1}$ and $2850 \mathrm{~cm}^{-1}$ are also present, they have a higher intensity and can be attributed to aldehyde $\mathrm{C}-\mathrm{H}$ groups and, most probable, to $\mathrm{H}-\mathrm{CF}_{2^{-}}$group.

In case of NAFION, the IR spectral analysis (Fig. 2) is also in accord with quantum chemical simulation. The characteristic bands, associated with vibration mode of C-O-C (980 and $\left.968 \mathrm{~cm}^{-1}\right)$ and $\mathrm{SO}_{3}\left(1320 \mathrm{~cm}^{-1}\right.$, $1202 \mathrm{~cm}^{-1}$ and $1060 \mathrm{~cm}^{-1}$ ), were decreasing at radiation doses between 100 and $1000 \mathrm{kGy}$. The high stability of PTFE backbone is also confirmed, C-F vibrations at $1200 \mathrm{~cm}^{-1}$ and $1144 \mathrm{~cm}^{-1}$ were unaffected.

The IR spectra of gamma-irradiated VITON sample (Fig. 3) show modification similar to that of irradiated PTFE. We have identified TFA characteristic bands $\left(1783 \mathrm{~cm}^{-1}\right.$ superposed on existing pick of reference sample, $\left.1447 \mathrm{~cm}^{-1}, 813 \mathrm{~cm}^{-1}\right)$. The backbone characteristic

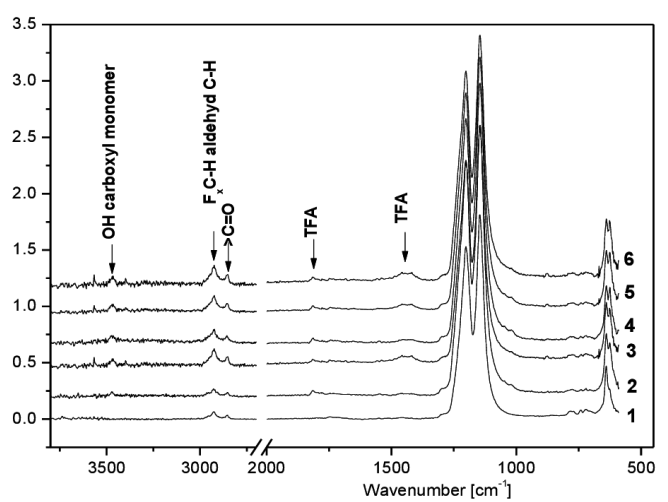

Fig. 1. IR spectra of unirradiated PTFE (1), gamma irradiated PTFE at $300 \mathrm{kGy}(2), 450 \mathrm{kGy}(3), 1000 \mathrm{kGy}$ (4) and PTFE exposed to HTO for 2 months (5) and 36 months (6).

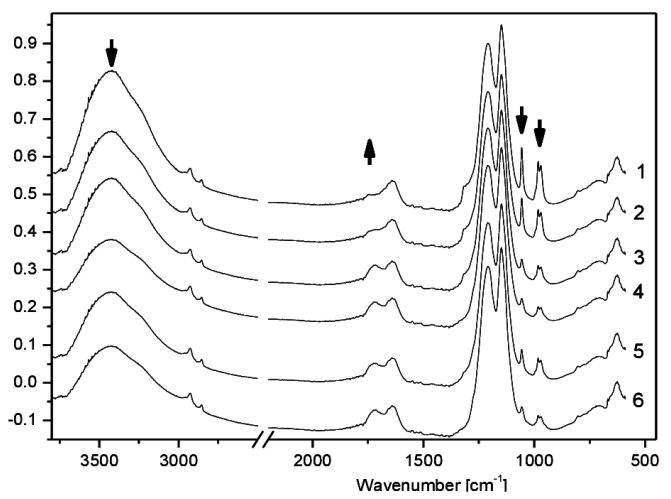

Fig. 2. IR spectra of unirradiated NAFION (1), gamma irradiated NAFION at $300 \mathrm{kGy}(2), 450 \mathrm{kGy}$ (3), $1000 \mathrm{kGy}(4)$ and NAFION exposed to HTO for 2 months (5) and 36 months (6).

twins pick at $1170-1126 \mathrm{~cm}^{-1}$ present a small modification at $1166-1129 \mathrm{~cm}^{-1}$ with the inversion of amplitude.

In case of samples exposed to high activity tritiated water, the IR spectra are similar to those of gammairradiated fluoropolymers at high absorbed dose.

\section{Conclusions}

The behaviour of PTFE, NAFON and VITON fluoropolymers, used in facilities for tritium recovery from HTO or DTO, was analyzed by following methods: simulation of radiolytic effects by computational methods, irradiation of samples immersed in water with a ${ }^{60} \mathrm{Co}$ gamma source, and by storage in tritiated water with high radioactive concentration for 60 and 182 days. Exposed samples were analyzed by IR spectrometry and fluoride release analysis.

In case of PTFE, used as isotope exchange support catalysts [2], quantum mechanical analysis shows evidence of main-chain homolytic fragmentation, associated with formation of $\mathrm{HF}$ and hydrophilic groups like $\mathrm{OH}$ and $\mathrm{COOH}$. Contribution of radio-induced hydrophilic 


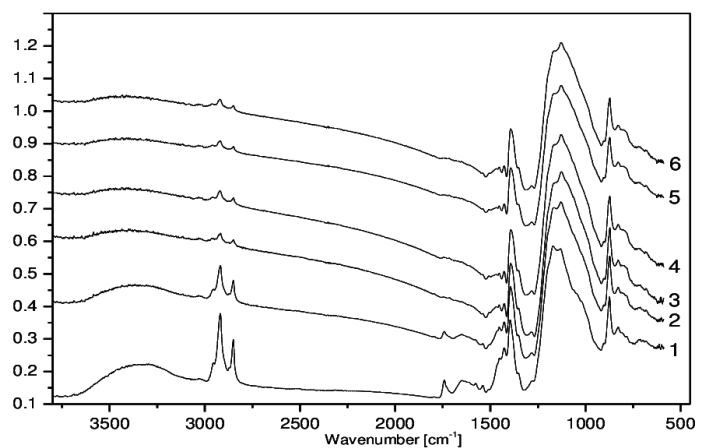

Fig. 3. IR spectra of unirradiated VITON (1), gamma irradiated VITON at $300 \mathrm{kGy}$ (2), $450 \mathrm{kGy}$ (3), $1000 \mathrm{kGy}(4)$ and VITON exposed to HTO for 2 months (5) and 36 months (6).

groups is insignificant and does not affect polymeric support properties. Formation of $\mathrm{HF}$ is, on the other hand, significant and cannot be ignored.

In NAFION, which is used as a solid polymer electrolyte, quantum chemical analysis evidenced a higher stability with respect to PTFE. Two tendencies for lots of hydrophilic groups were identified: fragmentation of $\mathrm{C}-\mathrm{SO}_{3}$ bonds and fragmentation of $\mathrm{C}-\mathrm{O}$ and $\mathrm{F}_{2} \mathrm{C}-\mathrm{CF}_{2}$ bonds from pendant hydrophilic sulfonate side chains. The fragmentation of every $\mathrm{F}_{2} \mathrm{C}-\mathrm{CF}_{2}$ bonds was associated with $\mathrm{HF}$ emission.

VITON fluoroelastomer, which is used as sealing material in tritium facilities, presents a higher stability to radiolysis with respect to $\mathrm{PTFE}$ and NAFION. The dominating radiolytic process is fragmentation of $\mathrm{CF}_{2}-\mathrm{CF}_{2}$ bonds from the main chain of the polymer, however the $G$ values obtained in the presented simulation were low.
Experimental radio-induced changes are evaluated by IR spectroscopy and fluoride release determination.

FTIR spectroscopy and fluoride release analyses are in accord with the predictions of quantum chemical simulation.

Obtained results suggest significant self-radiolytic processes in PTFE, NAFION and VITON fluoropolymers in the presence of tritiated water. In authors' opinion, fluoropolymers are not recommended to use as catalytic support, as electrolytic cell membranes and as sealing material in applications for detritiation of water or of the heavy water.

\section{Acknowledgments}

This researce is supported by the Project PNCDI II 177/2012 and Core Project No 09370206 from Executive Unit for Financing Education Higher Research Development and Innovation of Romania.

\section{References}

[1] I. Cristescu, et al., Fusion. Sci. Technol. 48, 97 (2005).

[2] Gh. Ionita, et al., Fusion. Sci. Technol. 48, 112 (2005).

[3] C. Postolache, L. Matei, Radiat. Phys. Chem. 76, 1267 (2007).

[4] L. Matei, et al., Fusion. Sci. Technol. 54, 643 (2008).

[5] L. Matei, C.Postolache, I.Cristescu, S. Brad, Fusion. Sci. Technol. 54, 475 (2008). 